Gut and Liver, Vol. 10, No. 2, March 2016, pp. 283-287

\title{
Evaluation of Liver Metastases Using Contrast-Enhanced Ultrasound: Enhancement Patterns and Influencing Factors
}

\author{
Wen-Tao Kong, Zheng-Biao Ji, Wen-Ping Wang, Hao Cai, Bei-Jian Huang, and Hong Ding \\ Department of Ultrasound, Zhongshan Hospital, Fudan University, Shanghai, China
}

Background/Aims: To evaluate the enhancement patterns of liver metastases and their influencing factors using dynamic contrast-enhanced ultrasound (CEUS). Methods: A total of 240 patients (139 male and 101 female; $58.5 \pm 11.2$ years of age) diagnosed with liver metastases in our hospital were enrolled in this study to evaluate tumor characteristics using CEUS. A comparison of enhancement patterns with tumor size and primary tumor type was performed using the chi-square test. The differences between quantitative variables were evaluated with the independent-sample t-test and one-way analysis of variance. Results: The enhancement patterns of liver metastases on CEUS were categorized as diffuse homogeneous hyperenhancement (133/240, 55.4\%), rim-like hyperenhancement $(80 / 240,33.3 \%)$, heterogeneous hyperenhancement (10/240, 4.2\%), and isoenhancement $(17 / 240,7.1 \%)$. There were significant differences in the enhancement patterns during the arterial phase based on the nodule size ( $p=0.001)$. A total of 231 of the nodules showed complete washout during the portal phase, and 237 nodules were hypoenhanced during the delayed phase. The washout time was correlated with tumor vascularity, with a longer washout time observed in hypervascular metastases compared to hypovascular metastases $(p=0.033)$. Conclusions: Diffuse homogeneous hyperenhancement followed by rapid washout was the most common enhancement pattern of liver metastases on CEUS and was affected by the nodule size and tumor vascularity. Small metastases were prone to show diffuse homogeneous hyperenhancement. Hypervascular metastases showed a significantly longer washout time compared to hypovascular metastases. (Gut Liver 2016;10:283-287)

Key Words: Contrast enhanced ultrasound; Liver metastases;
Enhancement pattern

\section{INTRODUCTION}

Metastasis is one of the most common liver malignancies. Patients with colorectal, breast, lung, and pancreatic cancers have a high incidence of liver metastases. ${ }^{1}$ Accurate diagnosis and assessment of nodules size, number and location is critical for the optimal therapeutic strategy, follow-up and prognosis. Conventional grey-scale ultrasound is usually regarded as the firststep imaging technique to evaluate liver metastases, though it is usually considered to be less accurate than enhanced computed tomography (CT) or magnetic resonance imaging (MRI). ${ }^{2}$ Nowadays, contrast enhanced ultrasound (CEUS) has been widely used in the evaluation of liver metastases with the advantage of dynamical detection of microcirculation within liver tumors. Most studies have found that CEUS improved both sensitivity and accuracy in the detection of metastases than conventional grey-scale ultrasound, especially for small nodules. ${ }^{3-5}$ Therefore, it is of great importance to be familiar with the characterization of liver metastases on CEUS. A rim-like enhancement during the arterial phase and early washout in the portal or delayed phase was once regard as typical findings of liver metastases on CEUS. ${ }^{6}$ However, some documents reported that CEUS appearances of liver metastases were related to the primary malignancy type. Hypervascular metastases were more frequent in neuroendocrine tumors, malignant melanoma and sarcoma, whereas hypovascular metastases usually occurred in patients with colorectal, gastric, pancreatic, or ovarian cancers. ${ }^{7}$ Besides, we also found the enhancement patterns variable, which could confuse the differential diagnosis.

The aim of this study was to characterize the CEUS patterns of liver metastases and analyze the influencing factors. On the

\footnotetext{
Correspondence to: Wen-Ping Wang

Department of Ultrasound, Zhongshan Hospital, Fudan University, Shanghai 200032, China

Tel: +86-21-64041990, Fax: +86-21-64220319, E-mail: puguang61@126.com

Received on August 19, 2014. Revised on February 6, 2015. Accepted on March 13, 2015. Published online November 23, 2015

pISSN 1976-2283 eISSN 2005-1212 http://dx.doi.org/10.5009/gnl14324

Wen-Tao Kong and Zheng-Biao Ji contributed equally to this work as first authors.

() This is an Open Access article distributed under the terms of the Creative Commons Attribution Non-Commercial License (http://creativecommons.org/licenses/by-nc/4.0) which permits unrestricted non-commercial use, distribution, and reproduction in any medium, provided the original work is properly cited.
} 
other hand, we aimed to investigate whether factors like tumor size or primary malignancy type may affect the enhancement patterns on CEUS.

\section{MATERIALS AND METHODS}

\section{Patient characteristics}

From January 2009 to March 2013, CEUS was performed in 240 patients (139 men and 101 women; mean age, 58.5 years; range, 16 to 88 years) with liver metastases in our hospital. The gold standard of the diagnosis of live metastases was pathological findings in the case of surgical resection or by percutaneous biopsy ( $n=66)$. Of the remaining cases, all had pathologically confirmed extrahepatic malignancies with liver metastases showing typical CT or MRI manifestations $(n=174)$ (rim-like enhancement and early wash-out). The primary malignancies included colorectal carcinoma in 121, lung cancer in 18, nasopharyngeal carcinoma in 12 , gastric carcinoma in 13 , pancreatic carcinoma in 19, neuroendocrine carcinoma in 12, breast carcinoma in 15, gastrointestinal stromal tumor in seven, ovarian cancer in eight, and other malignancies in 15 cases (sample size small than five for each disease) (Table 1).

First, liver metastases were classified into three groups according to nodules size: $\leq 20 \mathrm{~mm}, 21-50 \mathrm{~mm}$, and $>50 \mathrm{~mm}$. Each group then was divided into two subgroups according to tumor vascularity as reported by literature. ${ }^{7}$ Hypervascular metastases included neuroendocrine carcinoma $(n=12)$, breast carcinoma $(n=15)$, thyroid carcinoma $(n=2)$, malignant melanoma and sarcoma $(n=6)$, gastrointestinal stromal tumor $(n=7)$, and renal carcinoma $(n=1)$. Hypovascular metastases included colorectal carcinoma $(n=121)$, lung cancer $(n=18)$, nasopharyngeal carcinoma $(n=12)$, gastric carcinoma $(n=13)$, pancreatic carcinoma $(n=19)$, ovarian carcinoma $(n=8)$, esophagus carcinoma $(n=4)$, and gallbladder carcinoma $(n=2)$. Patients with history of allergic reaction or severe cardiac or pulmonary dysfunction

Table 1. Distribution of Primary Tumors of Liver Metastases

\begin{tabular}{lcc}
\hline \multicolumn{1}{c}{ Primary malignancy } & No. of cases & $\begin{array}{c}\text { Nodule size of liver } \\
\text { metastases, mm }\end{array}$ \\
\hline Colorectal carcinoma & 121 & $28.9 \pm 23.8$ \\
Lung cancer & 18 & $26.9 \pm 27.3$ \\
Nasopharyngeal & 12 & $24.2 \pm 15.8$ \\
Gastric carcinoma & 13 & $23.0 \pm 10.8$ \\
Pancreatic carcinoma & 19 & $27.9 \pm 16.2$ \\
Neuroendocrine & 12 & $28.3 \pm 23.8$ \\
Breast carcinoma & 15 & $24.3 \pm 13.7$ \\
Gastrointestinal stromal & 7 & $68.1 \pm 57.8$ \\
Ovarian cancer & 8 & $35.0 \pm 27.9$ \\
Other malignancies & 15 & $26.0 \pm 15.1$ \\
\hline
\end{tabular}

Data are presented as number or mean \pm SD. were excluded from this study. Written informed consent was obtained from all patients and the study was approved by the ethical committee of our institution.

\section{Contrast enhanced ultrasonography}

CEUS imaging was performed using LOGIQ E9 (GE, Chalfont St Giles, UK; $n=149$ ) and IU 22 (Philips, Bothell, WA, USA; $\mathrm{n}=91$ ) ultrasonic system. The probe frequency was set at 2 to $5 \mathrm{MHz}$, and the mechanical index was lower than 0.1. Before CEUS, the number, size, location and echo of nodules on greyscale ultrasound were recorded. A bolus of $2.4 \mathrm{~mL}$ of ultrasound contrast agent (UCA) (Sonovue; Bracco, Milan, Italy) was injected into antecubital vein followed by $5 \mathrm{~mL}$ of $0.9 \%$ normal saline. After injection of the microbubbles, the target tumor was first observed, followed by the whole liver scan in the delayed phase to find out any possible new hypoechoic nodules. If the CEUS image was not qualified, another $2.4 \mathrm{~mL}$ UCA was administrated to better observe the appearance on arterial phase. The observation time lasted for 5 minutes after injection. Patients were told to breathe quietly during examination. All CEUS examinations were recorded on magneto-optical disks for further analysis. The arterial phase is defined as from 10-20 to 30-45 seconds after injection. The portal phase followed until $120 \mathrm{sec}-$ onds after injection. And the delayed phase was defined as from 120 seconds after the injection until the clearance of the UCA from the circulation. ${ }^{8}$

\section{Imaging analysis}

The nodule appearances during arterial phase were defined as diffuse homogeneous hyperenhancement, rim-like hyperenhancement, diffuse heterogeneous hyperenhancement and isoenhancement. When the nodules appeared hyperechoic relative to adjacent liver parenchyma after injection, it was defined as initial enhancement. When the echo of nodules appeared lower than adjacent liver parenchyma, it was defined as hypoechoic and washout. The time to enhancement, time to peak and time to hypoechoic were recorded separately for further analysis. The timing was performed by subjective assessment of lesion brightness with the aid of the system timer or clock. CEUS imaging was retrospectively reviewed by two sonographers who have at least 5 years of CEUS experience to reach consensus.

\section{Statistical analysis}

Quantitative data were expressed as mean \pm standard deviation. A comparison of enhancement pattern with tumor size and primary tumor type was performed by using chi-square test. The differences between quantitative variables were evaluated with the independent-samples t-test and one-way analysis of variance test. $p$-values of $<0.05$ were considered to be statistical significant. All statistical analyses were performed using SPSS software version 17.0 (SPSS Inc., Chicago, IL, USA). 


\section{RESULTS}

Single nodules were detected in 122 patients and multiple in the remaining 118 patients. In patients with multiple nodules, only the largest one was selected as the target lesion to evaluate the enhancement pattern. A total of 240 nodules were analyzed in this study, with a mean diameter of $29 \pm 24.1 \mathrm{~mm}$ (range, 4 to $167 \mathrm{~mm}$ ) which is measured in the delayed phase on CEUS. The numbers of nodules $\leq 20 \mathrm{~mm}, 21-50 \mathrm{~mm}$, and $>50 \mathrm{~mm}$ were 114, 103, and 23, respectively.

On grey-scale ultrasound, the nodules were hypoechoic in 153 (63.8\%), hyperechoic in 68 (28.3\%), isoechoic in 14 (5.8\%), nonechoic in one $(0.4 \%)$, and mixed in the remaining four cases $(1.7 \%)$.

During the arterial phase, four enhancement patterns were recorded on CEUS: diffuse homogeneous hyperenhancement (133/240, 55.4\%), rim-like hyperenhancement (80/240, 33.3\%), heterogeneous hyperenhancement $(10 / 240,4.2 \%)$, and isoenhancement (17/240, 7.1\%). There were statistically significant differences in the enhancement pattern during arterial phase according the nodule size $(\mathrm{p}<0.001)$. Seventy-seven nodules smaller than $20 \mathrm{~mm}$ were diffuse homogeneous hyperenhanced (77/133, 57.9\%), with 54 nodules (54/133, 40.6\%) from 20 to 50 $\mathrm{mm}$ in size and two nodules (2/133, 1.5\%) larger than $50 \mathrm{~mm}$. However, there were no statistically significant differences in the enhancement pattern between hypervascular metastases and hypovascular metastases ( $\mathrm{p}=0.054)$.

The initial median time to enhancement and the median time to peak of the nodule were $17.3 \pm 4.3$ seconds (range, 8 to 38 seconds) and $22.4 \pm 5.2$ seconds (range, 11 to 47 seconds), respectively, with statistically significant differences between hypervascular and hypovascular metastases $(\mathrm{p}=0.001, \mathrm{p}=0.006)$.

Of all the nodules, 231 showed complete washout during portal phase. Nine nodules appeared isoechoic compared with the surrounding liver parenchyma (Fig. 1). In delayed phase, 237 nodules were hypoenhanced, whereas three were isoenhanced (medium time to washout, $43.3 \pm 33.8$ seconds; range, 19 to 300 seconds). Hypervascular metastases had a significantly longer washout time (53.5 \pm 54.3 seconds; range, 19 to 300 seconds) than hypovascular metastases $(41.2 \pm 27.3$ seconds; range, 19 to 270 seconds) ( $p=0.033$ ). However, there were no statistically significant differences between three groups of different nodule sizes in the washout time $(\mathrm{p}=0.131)$. The enhancement features of liver metastases and time of nodules enhancement after injection of SonoVue were detailed in Tables 2 and 3.
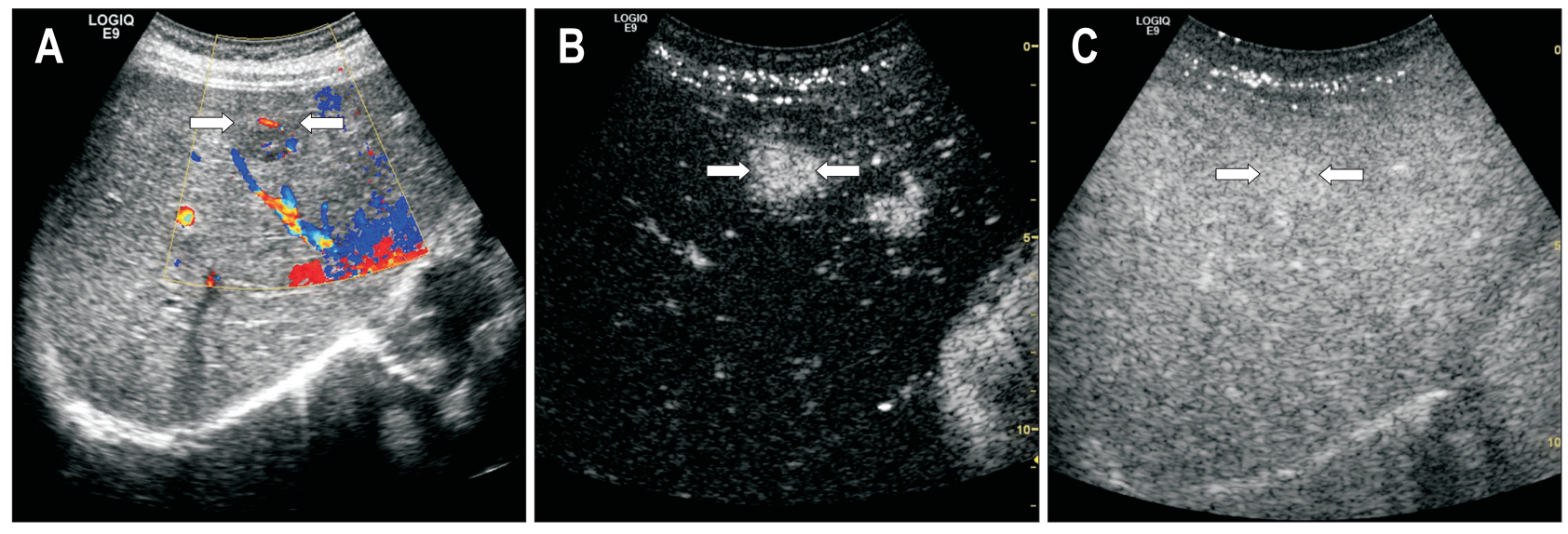

Fig. 1. Contrast-enhanced ultrasound of a 46-year-old man with pancreatic neuroendocrine carcinoma and liver metastases. (A) Conventional ultrasound showed a hypoechoic lesion (arrows) with blood flow. (B) The arterial phase showed homogeneous hyperenhancement of the lesion (arrows). (C) The lesion (arrows) remained isoechoic during the portal phase.

Table 2. Enhancement Pattern of Liver Metastases on Contrast-Enhanced Ultrasound according to Nodule Size

\begin{tabular}{cccccc}
\hline \multirow{2}{*}{$\begin{array}{c}\text { Nodule size, } \\
\text { mm }\end{array}$} & Nodule no. (\%) & $\begin{array}{c}\text { Enhancement pattern } \\
\text { hyperenhancement }\end{array}$ & $\begin{array}{c}\text { Rim-like } \\
\text { hyperenhancement }\end{array}$ & $\begin{array}{c}\text { Heterogeneous } \\
\text { hyperenhancement }\end{array}$ & Isoenhancement \\
\hline$<20$ & $114(47.5)$ & 77 & 25 & 0 & 12 \\
$20-50$ & $103(42.9)$ & 54 & 41 & 3 & 5 \\
$>50$ & $23(9.6)$ & 2 & 14 & 7 & 0 \\
Total & 240 & 133 & 80 & 10 & 17 \\
\hline
\end{tabular}

Data are presented as the number of nodules. 
Table 3. Enhancement Patterns and Time of Metastasis Enhancement in Terms of Nodule Blood Supply

\begin{tabular}{|c|c|c|c|c|}
\hline Enhancement pattern on CEUS & Group A & Group B & Total & $\mathrm{p}$-value \\
\hline Arterial phase & & & & 0.054 \\
\hline Homogeneous hyperenhancement & 105 & 28 & 133 & \\
\hline Rim-like hyperenhancement & 72 & 8 & 80 & \\
\hline Heterogeneous hyperenhancement & 6 & 4 & 10 & \\
\hline Isoenhancement & 14 & 3 & 17 & \\
\hline Portal phase & & & & $<0.001$ \\
\hline Hypoenhancement & 196 & 35 & 231 & \\
\hline Isoenhancement & 1 & 8 & 9 & \\
\hline Delayed phase & & & & 0.027 \\
\hline Hypoenhancement & 196 & 41 & 237 & \\
\hline Isoenhancement & 1 & 2 & 3 & \\
\hline No. of nodules & 197 & 43 & 240 & \\
\hline \multicolumn{5}{|l|}{ Time of nodule enhancement, sec } \\
\hline Initial time to enhancement & $17.7 \pm 4.3$ & $15.3 \pm 3.7$ & & 0.001 \\
\hline Time to peak enhancement & $22.8 \pm 5.0$ & $20.3 \pm 5.8$ & & 0.006 \\
\hline Washout time & $41.2 \pm 27.3$ & $53.5 \pm 54.3$ & & 0.033 \\
\hline
\end{tabular}

Group A includes hypovascular metastases, and group B includes hypervascular metastases. Data are presented as the number of nodules. CEUS, contrast-enhanced ultrasound.

\section{DISCUSSION}

Rim-like enhancement was once regarded as the characterization of liver metastases. However, the updated guidelines and recommendations for CEUS in the liver by The World Federation for Ultrasound in Medicine and Biology (WFUMB)-The European Federation of Societies for Ultrasound in Medicine and Biology (EFSUMB) pointed out metastases usually showed at least some contrast enhancement in the arterial phase and sometimes this was marked but often chaotic. ${ }^{7}$ There are some factors that may affect the enhancement patterns of liver metastases, such as primary malignancies with different biological behavior, pathological change along with tumor growth or discrepancy of imaging techniques.

Our study showed that diffuse homogeneous hyperenhancement, followed by rapid washout was the most common enhancement pattern of liver metastases. These results were in agreement with data from literatures. ${ }^{9}$ In our study, the enhancement pattern was correlated with nodules size, which may be resulted from the pathological change along with tumor growth. Small lesions tend to be homogeneously hyperenhanced for its abundance in tumor cells and vessels, whereas in large tumors, with intranodular necrosis or vascular thrombosis, the enhancement could be heterogeneous. Some authors described a more frequent rim-like enhancement pattern of hypovascular metastases, due to low arterial perfusion. ${ }^{8}$ These researches were usually based on CT or MRI. ${ }^{10}$ However, we found that in addition to hypervascular metastases, hyperenhancement could also be seen in some nodules which generally thought to be with poor tumor vascularity. CEUS can clearly show the continuous dynamic enhancement pattern of nodules, whereas CT or MRI images are usually acquired at a predetermined time point which could possibly miss the time of actual arterial hypervascularity. $^{9}$

There might be a confusion in the differential diagnosis between hepatocellular carcinoma (HCC) and some liver metastases with similar enhancement pattern, both of which could appear as "quickly wash in and out." However, HCCs often tend to present a relatively slower washout compared with metastases, especially for HCCs smaller than $3 \mathrm{~cm}$ or with better differentiation. ${ }^{11,12}$ Our study showed that washout within the initial 45 seconds occurred in 176 nodules and between 45 and 120 seconds in 48 nodules, which meant that the hyperenhancement was a transient process. The very early washout of liver metastases may be related to the relatively low volume of lesions compared to normal liver parenchyma, the effective venous drainage, the characterization of microvasculature, ${ }^{9}$ and also the absence of portal vein supply to the nodules. ${ }^{13,14}$ Unlike CT and MRI contrast agents, ultrasound contrast agents are exclusively intravascular. Therefore, the hypoenhancement may be observed during portal phase, which is helpful in the early detection of liver metastases.

Dănilă et al. ${ }^{7}$ thought the degree of arterial perfusion varied with the primary tumor type. In our study, although the proportions of enhancement patterns were not correlated with primary malignancy, we found the time to enhancement or washout may reflect the tumor vascularity of metastases. The time to initial enhancement and peak were correlated with tumor vas- 
cularity, with hypervascular nodules enhanced more quickly than hypovascular nodules. With regard to the time to hypoenhanced, hypervascular metastases appeared to wash out more slowly than hypovascular lesions. The media washout time of hypervascular metastases was 53.5 seconds, which was 41.2 seconds in hypovascular metastases. Five of the nine nodules showing isoechoic during portal phase were neuroen $\neg$ docrine tumor metastases, as well as three other nodules commonly thought to be hypervascular. This manifestation may be explained by the different tumor vascularity of metastases. With regard to hypervascular lesions, the increased arterial flow partially overlapped the reduced portal venous flow in the nodules, thus resulting prolonged arterial enhancement. ${ }^{15}$ However, hypovascular lesions could not compensate portal blood flow reduction due to fewer feeding vessels, thus displaying rapid washout on CEUS. Therefore, we postulated that the more tumor vascularity has the nodule, the longer the washout time will be. As a consequence, some hypervascular metastases may potentially mimic the appearance of benign focal liver lesions or welldifferentiated HCC. ${ }^{11}$ All the lesions may show isoenhancement during portal and delayed phase.

The major limitation of our study was the assessment of tumor vascularity was based on case history and literature. There was a lack of data on microvessel counts on pathologic specimens. Besides, we did not compare the differences between CEUS with CT or MRI in the characterization of metastases because the comparison of enhancement features of different imaging techniques was not the focus of our study. The small number of hypervascular nodules was another limitation in our study. There were few cases of certain tumors which may lead to selection bias.

In conclusion, transient homogeneous enhancement followed with rapid washout is the most common CEUS manifestation of liver metastases. Small metastases prone to show diffuse homogeneous hyperenhancement pattern, and hypervascular metastases had a significantly longer washout time than hypovascular metastases.

\section{CONFLICTS OF INTEREST}

No potential conflict of interest relevant to this article was reported.

\section{REFERENCES}

1. Wernecke K, Rummeny E, Bongartz G, et al. Detection of hepatic masses in patients with carcinoma: comparative sensitivities of sonography, CT, and MR imaging. AJR Am J Roentgenol 1991;157: 731-739.

2. Dietrich CF, Kratzer W, Strobe D, et al. Assessment of metastatic liver disease in patients with primary extrahepatic tumors by contrast-enhanced sonography versus CT and MRI. World J Gastroenterol 2006;12:1699-1705.

3. Piscaglia F, Corradi F, Mancini M, et al. Real time contrast enhanced ultrasonography in detection of liver metastases from gastrointestinal cancer. BMC Cancer 2007;7:171.

4. Correas JM, Low G, Needleman L, et al. Contrast enhanced ultrasound in the detection of liver metastases: a prospective multicentre dose testing study using a perfluorobutane microbubble contrast agent (NC100100). Eur Radiol 2011;21:1739-1746.

5. Sugimoto K, Shiraishi J, Moriyasu F, Saito K, Doi K. Improved detection of hepatic metastases with contrast-enhanced low mechanical-index pulse inversion ultrasonography during the liver-specific phase of sonazoid: observer performance study with JAFROC analysis. Acad Radiol 2009;16:798-809.

6. Claudon M, Cosgrove D, Albrecht T, et al. Guidelines and good clinical practice recommendations for contrast enhanced ultrasound (CEUS): update 2008. Ultraschall Med 2008;29:28-44.

7. Dănilă M, Popescu A, Sirli R, Sporea I, Martie A, Sendroiu M. Contrast enhanced ultrasound (CEUS) in the evaluation of liver metastases. Med Ultrason 2010;12:233-237.

8. Claudon M, Dietrich CF, Choi BI, et al. Guidelines and good clinical practice recommendations for contrast enhanced ultrasound (CEUS) in the liver-update 2012: a WFUMB-EFSUMB initiative in cooperation with representatives of AFSUMB, AIUM, ASUM, FLAUS and ICUS. Ultrasound Med Biol 2013;39:187-210.

9. Murphy-Lavallee J, Jang HJ, Kim TK, Burns PN, Wilson SR. Are metastases really hypovascular in the arterial phase? The perspective based on contrast-enhanced ultrasonography. J Ultrasound Med 2007;26:1545-1556

10. Kanematsu M, Kondo H, Goshima S, et al. Imaging liver metastases: review and update. Eur J Radiol 2006;58:217-228.

11. Loria F, Loria G, Basile S, Crea G, Randazzo D, Frosina L. Contrastenhanced ultrasound of hepatocellular carcinoma: correlation between enhancement pattern and cellular differentiation on histopathlogy. Updates Surg 2012;64:247-255.

12. Kong WT, Wang WP, Huang BJ, Ding H, Mao F. Value of wash-in and wash-out time in the diagnosis between hepatocellular carcinoma and other hepatic nodules with similar vascular pattern on contrast-enhanced ultrasound. J Gastroenterol Hepatol 2014;29: 576-580.

13. Cosgrove D, Blomley M. Liver tumors: evaluation with contrastenhanced ultrasound. Abdom Imaging 2004;29:446-454.

14. Larsen LP. Role of contrast enhanced ultrasonography in the assessment of hepatic metastases: a review. World J Hepatol 2010;2: 8-15.

15. Mörk H, Ignee A, Schuessler G, Ott M, Dietrich CF. Analysis of neuroendocrine tumour metastases in the liver using contrast enhanced ultrasonography. Scand J Gastroenterol 2007;42:652-662. 